\title{
On limits of Wireless Communications in a Fading Environment: a General Parameterization Quantifying Performance in Fading Channel
}

\author{
Amit Grover $^{1^{*}}$, Neeti Grover ${ }^{2}$ \\ $1^{1 *}$ Department of ECE, Shaheed Bhagat Singh State Technical Campus (SBSSTC), Ferozepur \\ ${ }^{2}$ Department of Applied Sciences, Shaheed Bhagat Singh State Technical Campus (SBSSTC), Ferozepur \\ e-mail: amitgrover321@gmail.com
}

\begin{abstract}
The reliable services along with high throughput can be achieved by using wireless communication systems. These systems also provides a wide coverage because of their features, no doubt MIMO Communication System [1] is one among them. Features provided by these systems ensure the improved system coverage and increased data transmission rate by considering multiple numbers of transmitter and receiver antennas. In this article, the concept of equalization has been considered and finally the performance of the MIMO Systems in Rician flat fading [5] channel is compared with the Rayleigh flat fading channel. It has also been observed that the performance of these Systems in Rician Flat Fading Channel is the best as compare to the Rayleigh Flat Fading Channel [10]. It has been concluded that the successive interference methods provide better performance as compare to others, but their complexity is high. Simulation results shows that ML provides the better performance in comparison to other equalizers but Sphere decoder provides the best performance.
\end{abstract}

Keywords: Quadrature Phase Shift Key (QPSK), Binary Phase Shift Key (BPSK), Minimum mean-squared error (MMSE), Maximum likelihood (ML), Successive linterference cancellation (SIC)

\section{Introduction}

The use of multiple antennas at the transmitter and receiver in wireless systems is known as MIMO Systems. Because of the features of MIMO systems, it became an important part of modern wireless communication [4]. MIMO-multiuser (MIMO-MU), refers to a configuration that comprises a base station with Multiple transmit/receive antennas interacting with multiple users [10]. Arrival of different transmitted signals through different angles results in multipath fading, which plays an important role in achieving the higher efficiency [11] in cellular and wireless communication [3].

\subsection{MIMO System Model}

By considering a MIMO system [4] with a transmit array of $\mathrm{M}_{\mathrm{T}}$ antennas and a receive array of $M_{R}$ antennas [8] as shown in Figure1. The transmitted matrix is a $M_{T} \times 1$ column matrix $s$ where $s_{i}$ is the ith component, transmitted from antenna $i$. We consider the channel to be a Gaussian channel, so that all the elements of $s$ are considered as Gaussian variables. If the channel is unknown at the transmitter, we assume that the signals transmitted from each antenna have equal powers of $E_{S} / M_{T}$. The covariance matrix for this transmitted signal is given by

$$
R_{S S}=\frac{E_{S}}{M_{T}} I_{M_{T}}
$$

Where $E_{S}$ is the power across the transmitter irrespective of the number of antennas $M_{T}$ and $I_{M T}$ is an identity matrix. The channel matrix $H$ is $M_{R} X M_{T}$ complex matrix. The component $h_{i, j}$ of the matrix is the fading coefficient from the jth transmit antenna to the ith receive antenna. We assume that the received power for each of the receive antennas is equal to the total transmitted power. The received signals constitute a $M_{R} \times 1$ column matrix denoted by $r$, where each complex component refers to a receive antenna. Since we assumed that the total received power per antenna is equal to the total transmitted power, the SNR can be written as 


$$
\mathrm{Y}=\mathrm{E}_{\mathrm{S}} / \mathrm{N}_{0}
$$

Where $E_{S}$ is the signal power and $N_{0}$ is the noise power. Let us consider $2 \times 2$ systems. The received signal on the first receive antenna is

$$
r_{1}=h_{11} s_{1}+h_{12} s_{2}+n_{1}
$$

And the received signal on the second receive antenna is

$$
r_{2}=h_{21} s_{2}+h_{22} s_{2}+n_{2}
$$

Where $r_{1}$ and $r_{2}$ are the received symbol on the first and second antennas respectively, $h_{11}$ is the channel from $1^{\text {st }}$ transmit antenna to $1^{\text {st }}$ receive antenna, $h_{12}$ is the channel from $2^{\text {nd }}$ transmit antenna to $1^{\text {st }}$ receive antenna, $h_{21}$ is the channel from $1^{\text {st }}$ transmit antenna to $2^{\text {nd }}$ receive antenna, $h_{22}$ is the channel from $2^{\text {nd }}$ transmit antenna to $2^{\text {nd }}$ receive antenna, $s_{1}, s_{2}$ are the transmitted symbols and $\mathrm{n}_{1}, \mathrm{n}_{2}$ is the noise on $1^{\text {st }}$ and $2^{\text {nd }}$ antennas respectively.

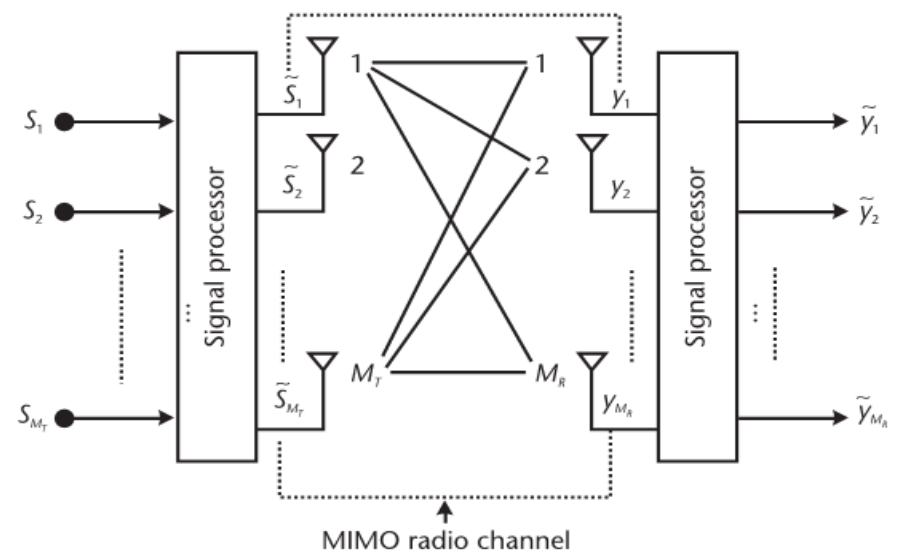

Figure 1. MIMO system model

Equation (3) and (4) can be represented in matrix form as

$$
\left[\begin{array}{l}
r_{1} \\
r_{2}
\end{array}\right]=\left[\begin{array}{ll}
h_{11} & h_{12} \\
h_{21} & h_{22}
\end{array}\right]\left[\begin{array}{l}
s_{1} \\
s_{2}
\end{array}\right]+\left[\begin{array}{l}
n_{1} \\
n_{2}
\end{array}\right]
$$

Therefore, the received vector can be expressed as

$$
r=H s+n
$$

For a system with $M_{T}$ transmit antennas and $M_{R}$ receive antennas, the MIMO channel at a given time instant may be represented as a $M_{T} \times M_{R}$ matrix

$$
\mathrm{H}=\left[\begin{array}{cccc}
\mathrm{H}_{1,1} & \mathrm{H}_{1,2} & \cdots & \mathrm{H}_{1, \mathrm{M}_{\mathrm{T}}} \\
\mathrm{H}_{2,1} & \mathrm{H}_{2,2} & \cdots & \mathrm{H}_{2, \mathrm{M}_{\mathrm{T}}} \\
\vdots & \vdots & \ddots & \vdots \\
\mathrm{H}_{\mathrm{M}_{\mathrm{R}}, 1} & \mathrm{H}_{\mathrm{M}_{\mathrm{R}}, 2} & \cdots & \mathrm{H}_{\mathrm{M}_{\mathrm{R}}, \mathrm{M}_{\mathrm{T}}}
\end{array}\right]
$$

\subsection{MIMO Channel}

Rayleigh fading channel model

By considering an extreme condition where all antenna elements at the transmitter are collocated and likewise at the receiver, all the elements of $\mathrm{H}$ will be fully correlated [2]. De- 
correlation between the channel elements will increase with antenna spacing. Scattering in the environment in combination with adequate antenna spacing ensures de-correlation of the MIMO channel elements [6].

Real- World MIMO channel

The presence of a fixed (possibly line-of-sight or LOS) component in the channel will result in Ricean fading [9]. In the presence of an LOS component between the transmitter and the receiver, the MIMO channel may be modeled as the sum of a fixed component and a fading component [3].

\section{Equalization}

\subsection{Zero Forcing}

An ISI channel may be modeled by an equivalent finite-impulse response (FIR) filter plus noise. A zero-forcing equalizer uses an inverse filter to compensate for the channel response function [7]. By assuming $M_{T}=M_{R}$ and $H$ is a full rank square matrix, we can calculate the covariance matrix of the effected noise as:

$$
E\left[\left(n \mathrm{H}^{-1}\right)^{H} \cdot n \mathrm{H}^{-1}\right]=\left(H^{-1}\right)^{H} \cdot E\left[n^{H} \cdot n\right] \cdot H^{-1}=n\left(H \cdot H^{H}\right)^{-1}
$$

\subsection{Minimum Mean Square Error (MMSE)}

By assuming that the number of receive antennas is less than the number of transmits antennas, MMSE at a high SNR is given by:

$$
W_{M M S E}=\left(H^{*} H+\frac{1}{S N R} I\right)^{-1} H^{*} \approx\left(H^{H} H\right)^{-1} H^{H}
$$

\subsection{Successive Interference Cancelation}

In this, the outputs of previous detectors are used that leads to decision detecting algorithms like Parallel Interference cancelation (PIC) and SIC. After the detection of first bit by the decorrelator the result is used to cancel the interference from the received signal vector assuming the decision of the first stream is correct [2].

\subsection{Maximum Likelihood (ML)}

The ML method achieves the optimal performance as the maximum a posterior detection when all the transmitted vectors are likely [2].

\subsection{Sphere Decoder (SD)}

Limited number of codeword's is considered in sphere decoder that is within a sphere centered at the received signal vector. In other words, the ML solution vector is now constrained in this sphere with a reduced radius [9]. By using this technique we find the ML solution vector that considers only a small set of vectors within a given sphere rather than all possible transmitted signal vectors [2].

\section{Simulation and Results}

By using MATLAB, the different simulation results are shown in the different graphs, which provide the comparison of the BER for different modulation techniques using different equalizers with Rician Flat Fading [5] channel. 


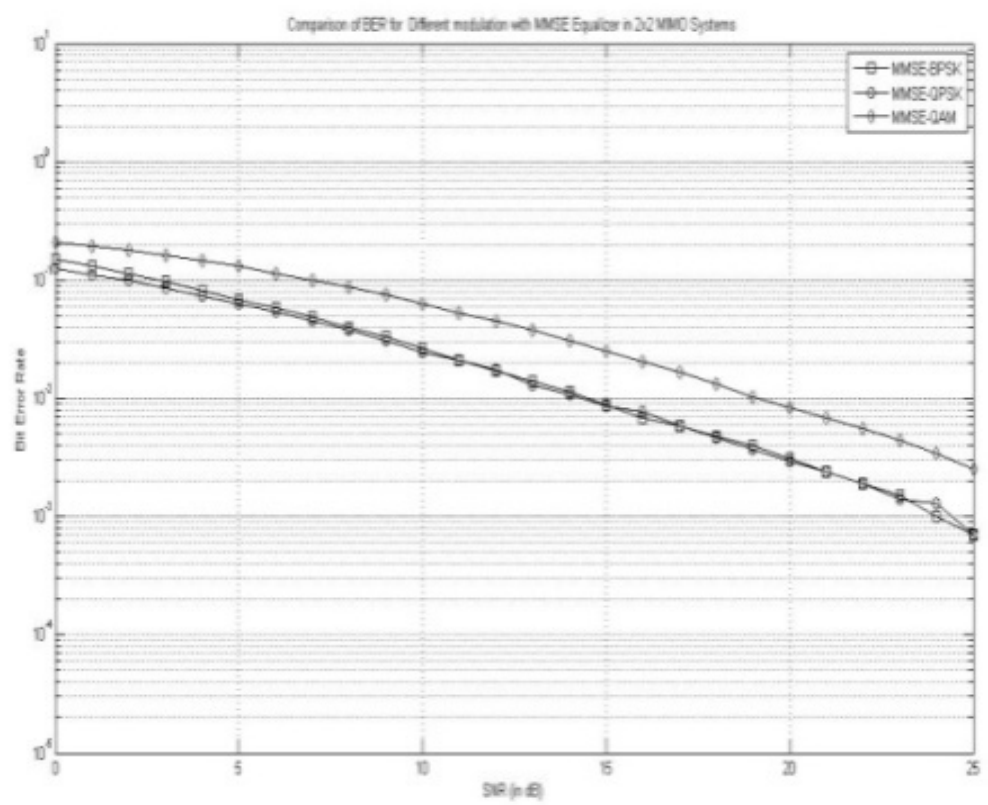

Figure 1. Comparison of BER for different modulation with MMSE equalizer in 2 x2 MIMO systems in Rician Flat Fading Channel

Figure 1 shows the simulation results over two transmit and two receive antennas using BPSK, QPSK and 16 QAM. It has been observed from Figure 1, the simulated values for BER at 10dB SNR are, for BPSK 0.1092, for QPSK 0.1266 and for 16 QAM 0.2616 which shows the better result of BPSK than QPSK and 16QAM.

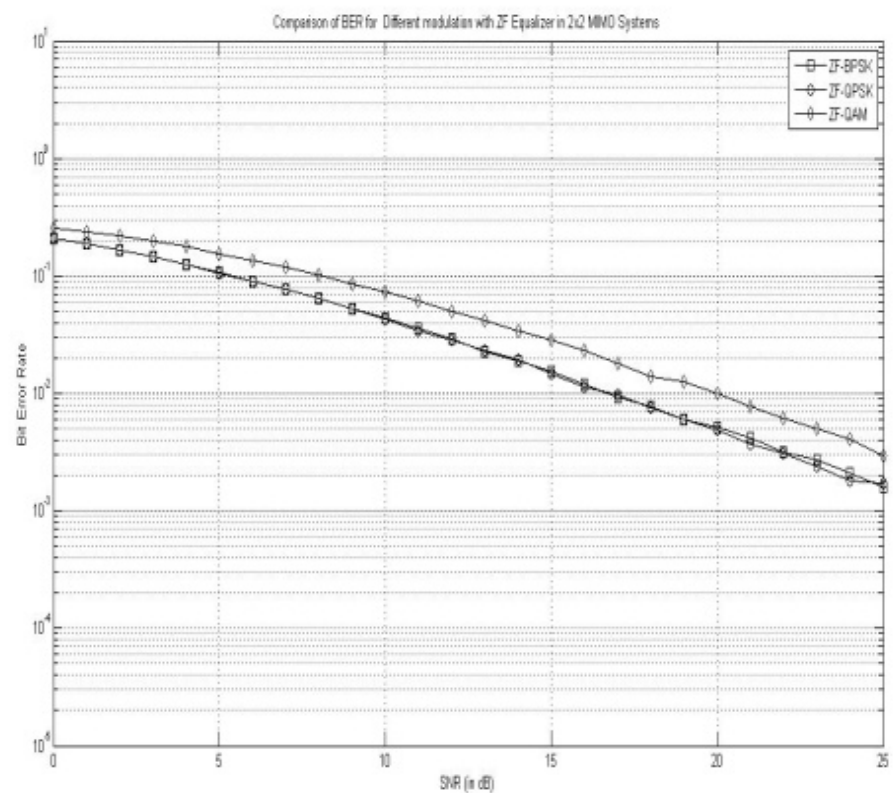

Figure 2. Comparison of BER for different modulations with ZF equalizer in $2 \times 2 \mathrm{MIMO}$ systems in Rician Flat Fading Channel 
Figure 2 shows the simulation results over two transmit and two receive antennas using BPSK, QPSK and 16 QAM. It has been observed from Figure 2, at $10 \mathrm{~dB}$ SNR, the BER for BPSK is 0.2148 , for QPSK is 0.2152 and for 16 QAM is 0.3092 which shows the better result of BPSK than QPSK and 16QAM.

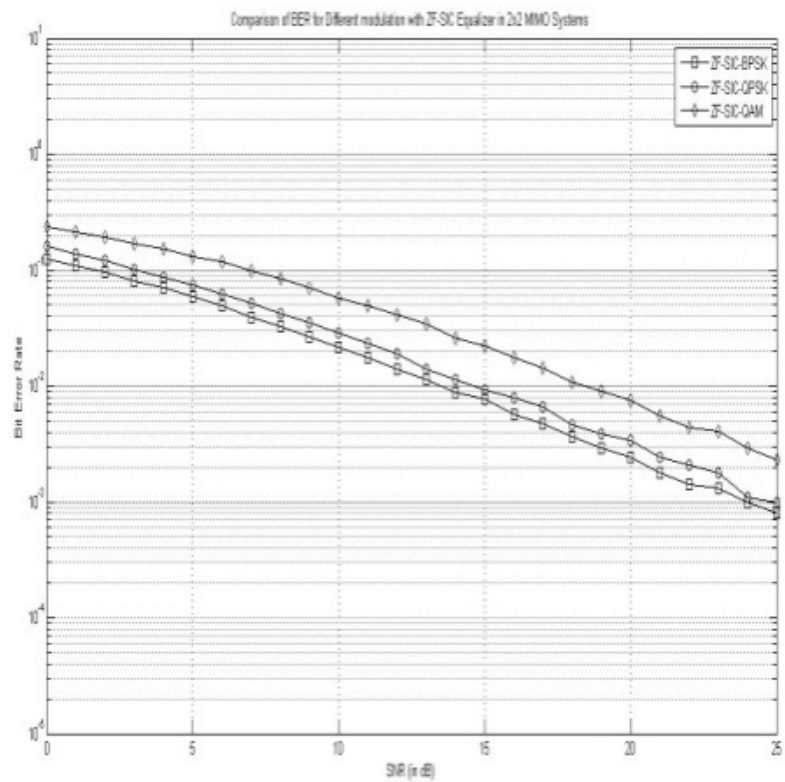

Figure 3. Comparison of BER for different modulations with ZF-SIC equalizer in $2 \times 2 \mathrm{MIMO}$ systems in Rician Flat Fading Channel

Figure 3 shows the simulation results over two transmit and two receive antennas using BPSK, QPSK and 16 QAM. It has been observed from Figure 3, the simulated values for BER at $10 \mathrm{~dB}$ SNR are for BPSK 0.1178, for QPSK 0.1472 and for 16QAM 0.2646 which shows the better result of BPSK than QPSK and 16QAM.

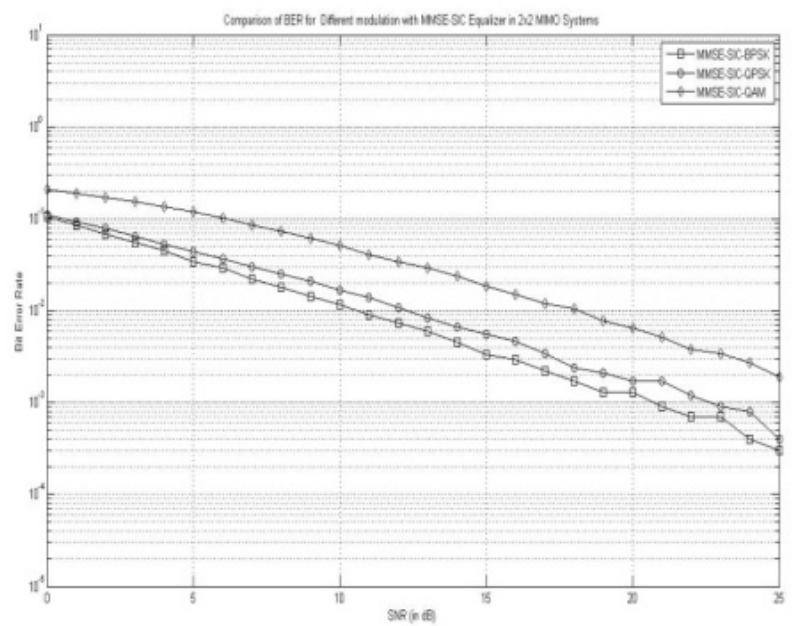

Figure 4. Comparison of BER for different modulations with MMSE-SIC equalizer in $2 \times 2$ MIMO systems in Rician Flat Fading Channel 
From Figure 4, it has been observed that the BER performance of MMSE-SIC is better than previous discussed equalizers. At $10 \mathrm{~dB}$ SNR, BER for BPSK is 0.0688 , for QPSK is 0.0886 and for 16 QAM 0.2358 .

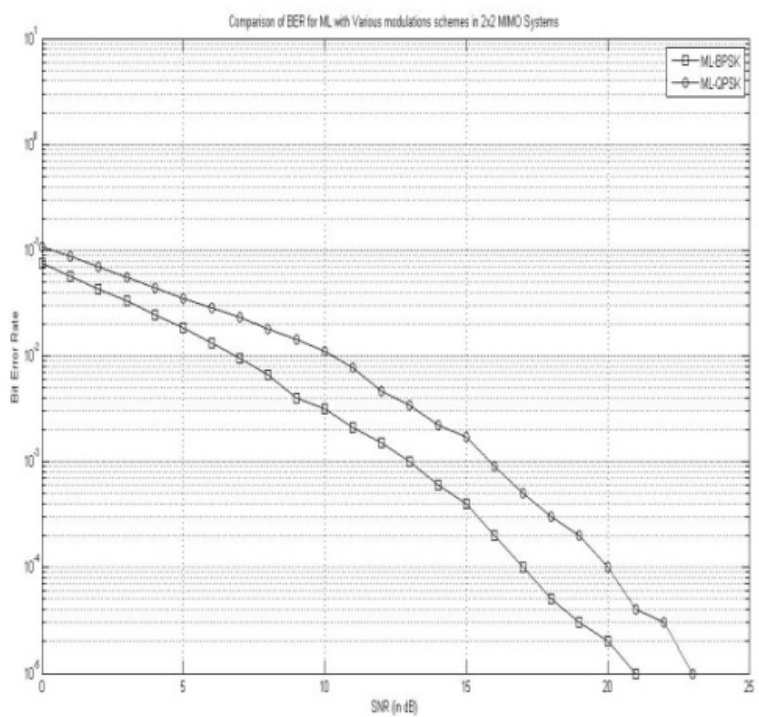

Figure 5. Comparison of BER for different modulations with ML equalizer in $2 \times 2 \mathrm{MIMO}$ systems in Rician Flat Fading Channel

From Figure 5, it has been observed that the complexity of $\mathrm{ML}$ equalizer increases as we move from BPSK to QPSK. At $10 \mathrm{~dB}$ SNR, BER for BPSK modulation is observed as 0.037 and BER for QPSK modulation is 0.0698 .

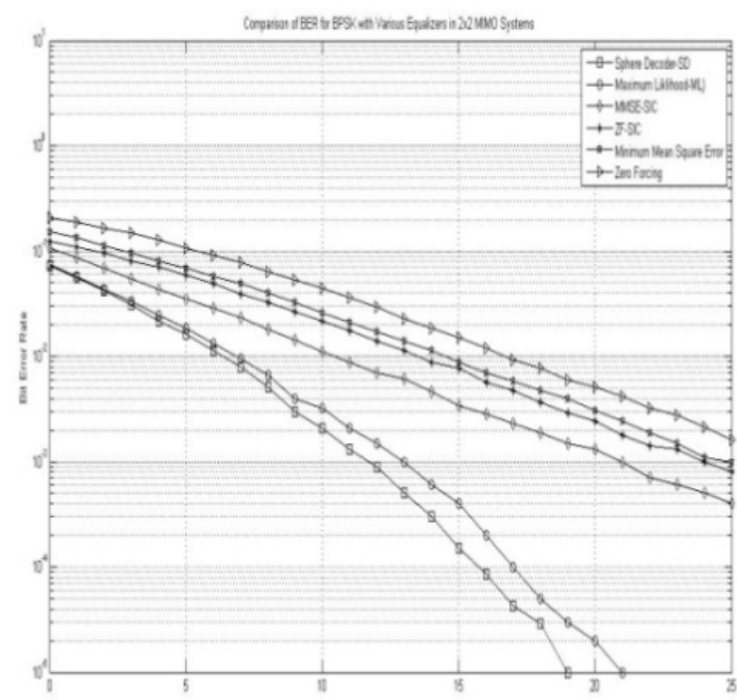

Figure 6. Comparison of BER for BPSK with different equalizers in $2 \times 2 \mathrm{MIMO}$ systems in Rician Flat Fading Channel 
Figure 6 shows the simulation results over two transmit and two receive antennas using BPSK. The results are decoded using the ZF, MMSE, ZF-SIC, MMSE-SIC, ML and Sphere decoder (SD) technique. The Simulated values for BER at $10 \mathrm{~dB}$ SNR are observed as for SD is 0.0326 , for ML is 0.037 , for MMSE-SIC 0.0698 , for ZF-SIC 0.1178 , for MMSE 0.1374 and for ZF $B E R$ is 0.2150 . So we concluded that the linear equalizers perform worse than other methods while requiring a lower complexity. The successive interference methods outperform the ZF and MMSE however their complexity is higher due to iterative nature of the algorithms. ML provides the better performance in comparison to all previously discussed. Sphere decoder provides the best performance and the highest decoding complexity as compare to ML.

\section{Conclusion}

In this article, the equalization through Rician Flat Fading Channel has been considered and the better results have been found as compared to Rayleigh flat fading Channel [3]. In Rician Flat Fading Channel the Performance of $M L$ is better than other equalizers but the the sphere decoder provides the best performance. The complexity of $\mathrm{ML}$ decoder increases as we move to higher modulation schemes, whereas complexity in SD depends on how well the initial radius is chosen. BER performance in the order of highest to lowest is as: SD > ML > MMSESIC > ZF-SIC > MMSE > ZF. Finally, it has been concluded that SD is the best suited method in Rician Flat Fading Channel in MIMO Systems.

\section{References}

[1] H El Gamal and AR Hammons. "The layered space-time architecture: a new perspective". IEEE Trans. Inform. Theory. 2001; 47: 2321-2334.

[2] GJ Foschini and MJ Gans. "On limits of wireless communications in a fading environment when using multiple antennas". Wireless Personal Communications. 1998; 6: 311-335.

[3] GJ Foschini. "Layered space-time architecture for wireless communications in a fading environment when using multiple antennas". Bell Labs. Tech. Journal. 1996; 6(2): 41-59.

[4] M Janakiraman. "Space-time codes and MIMO systems". Artech House. 2004.

[5] Wang and GB Giannakis. "A simple and general parameterization quantifying performance in fading channels". IEEE Trans. Commun. 2003; 51(8): 1389-1398.

[6] Ezio Biglieri, Robert Calderbank, Anthony Constantinides, Andrea Goldsmith, Arogyaswami Paulraj, H. Vincent Poor. "MIMO Wireless Communications". Cambridge University Press. 2007.

[7] G Arslan, BL Evans, and S Kiaei. "Equalization for Discrete Multitone Receivers To Maximize Bit Rate". IEEE Transactions on Signal Processing. 2001; 49(12): 3123-3136.

[8] D Shiu, PJ Smith, D Gesbert, M Shafi and A Nayguib. "From theory to practice: An overview of MIMO space-time coded wireless systems". IEEE Journal of Selected Areas in Communication. 2003; 21(3): $281-302$.

[9] Sergey Loyka and Francois Gagnon. Senior Member, IEEE," Performance Analysis of the V-BLAST Algorithm: An Analytical Approach". IEEE Transactions on Wireless Communications. 2004; 3(4): 1326-1336.

[10] L Zheng and D Tse. "Diversity and multiplexing: A fundamental trade-off in multiple-antenna channels". IEEE Transactions on Information Theory. 2003; 49: 1073-1096.

[11] Gerard J Foschini, Glen D Golden, Reinaldo A Valenzuela, Fellow, IEEE, and Peter W Wolniansky. "Simplified Processing for High Spectral Efficiency Wireless Communication Employing Multi-Element arrays". 1999; 17(11): 1841-1852. 